\title{
STREPTOMYCIN-DEPENDENT VARIANTS OF BACT. COLI ISOLATED FROM PATIENTS DURING TREATMENT WITH STREPTOMYCIN
}

BY

\author{
AKSEL STENDERUP* \\ From the Institute for General Pathology, University of Aarhus, Denmark
}

(RECEIVED FOR PUBLICATION AUGUST 17, 1953)

Since the publication in 1947 of Miller and Bohnhoff's report on streptomycin-dependent meningococci, streptomycin-dependent variants of many other bacterial species have been isolated and studied in vitro (Paine and Finland, 1948 ; Kushnick, Randles, Gray, and Birkeland, 1947 ; Yegian, Budd, and Vanderlinde, 1949). Miller and Bohnhoff also showed streptomycin-dependence in vivo when mice infected with streptomycin-dependent meningococci survived unless they were treated with streptomycin.

Reports on the development of streptomycindependent variants observed clinically seem to have been relatively scanty. Miller and Bohnhoff found streptomycin-dependent bacteria in the throats of patients who had been treated with streptomycin, but these microorganisms did not play any role in the pathogenesis of the patients' illnesses. Dufourt, Brun, Viallier, and Kalb (1951) found streptomycin-dependent tubercle bacilli in patients receiving streptomycin treatment and claimed that continued administration of streptomycin in certain cases aggravated the infection.

Eight cases of infection of the urinary tract treated with streptomycin are reported. During the treatment bacterial flora emerged which were only able to multiply on media containing streptomycin, i.e., the cultures were completely streptomycin dependent. After the withdrawal of streptomycin the streptomycin-dependent bacteria disappeared. In one patient renewed administration of streptomycin resulted in aggravation of the infection. This case will be described in detail together with a brief account of the seven other cases.

\section{Personal Investigations}

Case 1.-A woman, aged 78, who had suffered from recurrent cystitis for many years, was admitted to hospital on August 29, 1952, with a diagnosis of encephalomalacia and cystitis. On admission she

* Lady Tata memorial scholar. complained of burning micturition. Microscopic examination of the urine showed a few leucocytes, and urinary cultures yielded growth of numerous colonies of Gram-negative, lactose-fermenting rods with a sensitivity (determined by the paper-disc method (K. A. Jensen) ) to streptomycin +++ , sulphathiazole 0 , aureomycin + , chloromycetin + , terramycin ++ . Dihydrostreptomycin, 0.5 g., was administered four times daily for four days. On the day when streptomycin was withdrawn urinary cultures yielded growth of numerous colonies of Gram-negative lactose-fermenting rods, which only grew on that particular part of the "resistance plate" where the streptomycin disc had been placed. When the bacteria from the resistance plate and from the urine were seeded on agar-plates with and without streptomycin growth only occurred on the plates containing streptomycin. The urine contained a larger number of leucocytes than at the institution of treatment, even though the symptoms had subsided.

The urine was then studied daily, and for 11 days in succession only such bacteria which were able to grow on streptomycin-containing media were found. After that time the flora changed and now chiefly consisted of streptomycin-sensitive bacteria, although a few colonies of streptomycin-dependent organisms could still be isolated. This phenomenon was easily recognized on the resistance plate. On the part where the streptomycin was deposited there was a broad zone of inhibition in the area in which a few colonies grew, and when these were transferred to agar they did not form colonies unless streptomycin was added to the medium.

By way of experiment, the patient was then again given streptomycin, $0.25 \mathrm{~g}$., four times daily for one day. Five hours after the first streptomycin injection the bacterial flora had changed, and now again consisted exclusively of streptomycin-dependent bacteria. The next day the urine contained 50-100 leucocytes per field, which was interpreted as an activation of the infection, although no subjective symptoms developed. For six days the bacterial flora were streptomycin-dependent and then changed once more, so that it now mainly consisted of streptomycin-sensitive bacteria and only a few streptomycin-dependent organisms. 
A previous examination of the faecal flora for streptomycin-dependent bacteria had been negative. In order, if possible, to provoke a change in the faecal flora, the patient was given an oral dose of $1 \mathrm{~g}$. of streptomycin daily for three days. Twenty-four hours after the first oral dose the urinary flora had again become streptomycin dependent, and the number of leucocytes in the urine had increased very considerably, myriads of leucocytes per field being present, which had not previously been observed. At the same time, the patient complained of increased frequency of micturition, but there was no pain on micturition. In the faeces the number of coliform bacteria showed a marked decrease during the oral streptomycin treatment, but it was not possible to isolate streptomycin-dependent organisms. This seems to indicate that some factor in the urine was of importance in the change of the bacterial flora to streptomycin dependence.

The patient was discharged and returned for followup examination three months later. During the interval there had been no difficulty in micturition. The urine still contained Gram-negative, lactose-fermenting rods, but these were now streptomycin resistant, and no streptomycin-dependent organisms could be isolated.

This case illustrates the fact that flora of streptomycin-dependent bacteria may be found in the urine when urinary infections caused by streptomycin-sensitive organisms are treated with streptomycin. It also shows that the infection may be aggravated when streptomycin treatment is continued. This is in agreement with results from animal experiments performed with the strain which had been isolated in the above-mentioned case. Mice infected with the strain did not die unless they were treated with streptomycin (Stenderup, 1954).

It is remarkable that the urine contained completely streptomycin-dependent bacteria for as long as 11 days after streptomycin had been withdrawn, and at a time when no measurable amounts of streptomycin were detectable in the urine. However, when the dependent strain was transferred to plain broth and incubated at $37^{\circ} \mathrm{C}$., it did not change its streptomycin dependence, but remained alive in the broth for eight days after which time the broth became sterile.

Case 2.-A woman, aged 55, who had had several acute attacks of urinary infection during the past 15 years, was admitted to hospital on July 23,1948 , with a diagnosis of chronic pyelonephritis caused by streptomycin-sensitive, lactose-fermenting, Gram-negative rods. Streptomycin was administered, $500 \mathrm{mg}$., four times a day during the first 24 hours, followed by $0.3 \mathrm{~g}$. daily for the next five days. Four days later the urinary cultures showed that the bacteria had all become completely streptomycin dependent. Two days after the withdrawal of streptomycin cultures yielded growth of Gram-negative, lactose-fermenting rods, which were not dependent on streptomycin, but had become sensitive to streptomycin.

Case 3.-A woman, aged 32, without any past history of previous urinary infections was admitted to hospital on October 28, 1949, for vaginal bleeding during pregnancy. During the hospital stay a urinary infection developed caused by streptomycin-sensitive, lactose-fermenting, Gram-negative rods. Streptomycin was administered, $2 \mathrm{~g}$., during the first 24 hours, followed by $1 \mathrm{~g}$. daily, given in four doses. During the treatment the fever subsided and the subjective symptoms disappeared. After four days' treatment streptomycin was discontinued and on the same day urinary cultures yielded numerous colonies of Gramnegative, lactose-fermenting rods which were all dependent on streptomycin. Repeated cultures three days later revealed a different bacterial flora. No Gram-negative rods but numerous colonies of Grampositive cocci were seen.

Case 4.-A woman, aged 50, with a past history of recurrent urinary infections extending over many years was admitted to hospital on April 23, 1950, with a complaint of difficulty in micturition. Urinary cultures yielded growth of streptomycin-sensitive, lactose-fermenting, Gram-negative rods for which reason streptomycin was administered, $0.5 \mathrm{~g}$., thrice daily for six days. Urinary cultures performed on the day when streptomycin was withdrawn yielded growth of numerous colonies of Gram-negative, lactose-fermenting rods, which were all streptomycin dependent. Three days later urinary cultures showed innumerable colonies of Gram-negative, lactose-fermenting rods which were now resistant to streptomycin.

Case 5.-A man, aged 74, for seven years had had recurrent urinary infections. The patient was admitted to hospital on January 21, 1951, in an acute attack caused by streptomycin-sensitive, lactose-fermenting, Gram-negative rods. The patient was treated with streptomycin, $1 \mathrm{~g}$. daily, for five days, when renewed urinary cultures showed lactose-fermenting rods which were now streptomycin dependent. Streptomycin was then withdrawn, but after the lapse of another five days there was still growth of streptomycin-dependent, Gram-negative rods and Gram-positive cocci, which were not dependent on streptomycin. As the Gram-positive cocci were sensitive to aureomycin, the patient was treated with this drug, and the urine became sterile. During the streptomycin therapy the patient became symptom-free.

Case 6.-A woman, aged 31, who had had repeated attacks of urinary infections for about 14 months, was admitted to hospital on October 29, 1951, in an acute attack caused hy streptomycin-sensitive, lactose-fermenting, Gram-negative rods. The patient was treated with streptomycin, which resulted in a fall in the temperature and improvement of the general condition. Renewed urinary cultures after eight days' 
treatment showed growth of some colonies of lactosefermenting rods which were streptomycin dependent, and the same was the case two days after withdrawal of streptomycin. The patient was discharged and not observed for the next two months when the urine contained streptomycin-resistant, lactose-fermenting, Gram-negative rods.

Case 7.-A woman, aged 50 , with a past history of good health, except for " renal gravel" when a child, was admitted to hospital on November 20, 1951, with fever and arthritic pain.

The patient was treated with penicillin and later with aureomycin without any definite beneficial effect. Four days after the discontinuance of the aureomycin therapy the patient . began to complain of burning micturition, and the urine, which had previously been normal, now revealed numerous leucocytes and Gramnegative rods which were streptomycin sensitive. Streptomycin was then administered, $0.5 \mathrm{~g}$., four times daily for eight days. During the treatment the urinary symptoms subsided, but in the urine there were still many leucocytes and bacteria, which proved to be Gram-negative, streptomycin-dependent rods. A week after the withdrawal of streptomycin there was still growth of streptomycin-dependent, lactose-fermenting, Gram-negative rods. Then the flora changed to non-lactose-fermenting rods, which were sensitive to chloromycetin, and after treatment with this drug the urine became sterile and the microscopic findings normal.

Case 8.-A woman, aged 37, without any past history of urinary infections, had repeatedly been admitted to hospital with a diagnosis of tuberculous salpingitis and for observation for tuberculous peritonitis. During a hospital stay in 1950 a routine examination of the urine revealed growth of colonies of Gram-negative, lactose-fermenting rods, which showed more abundant growth on a streptomycincontaining medium than on media without streptomycin. The culture was thus only partially dependent on streptomycin and differed from the cultures isolated from Cases 1-7, which did not grow at all when seeded on streptomycin-free media. The patient showed no signs of disease of the urinary tract. At the time when this was disclosed, the patient was under treatment with streptomycin. During the period immediately following the urine was not subjected to renewed examination.

Nine months later an acute attack of cystitis developed; urinary cultures revealed Gram-negative, non-lactose-fermenting rods. These organisms were not dependent on streptomycin, and at that time the patient was not under treatment with streptomycin.

Case 8 shows that streptomycin-dependent bacteria may temporarily emerge in the urine during streptomycin therapy which does not aim at combating a urinary-tract infection.
In Cases 2 to 7 streptomycin-dependent flora emerged in the urine during treatment of urinary infections caused by streptomycin-sensitive bacteria. In contrast to Case 1 it could not be said with certainty that the dependent flora caused an aggravation of the infection, except perhaps in Case 7 , in which the urine contained myriads of leucocytes together with the streptomycin-dependent bacteria. Out of regard for the patients streptomycin treatment was not resumed, but it is possible that an aggravation might have occurred if this had been done. At any rate this assumption seems to be in accordance with the results of animal experiments.

When the strains were inoculated intraperitoneally together with mucin into white mice the animals survived, unless they were treated with streptomycin.

In one case (Case 3) the streptomycin-dependent, Gram-negative rods in the urine disappeared completely. In the other cases Gram-negative rods persisted, but changed their behaviour to streptomycin.

In Case 3 the bacteria changed to streptomycin sensitivity, in Cases 1, 4, 6 to streptomycin resistance. This is in accordance with in vitro experiments which were performed on the isolated streptomycin-dependent strains. These experiments showed that when a sufficiently large inoculum was seeded in a streptomycin-free medium, growth of bacteria developed. Some of these cultures were resistant, while others were sensitive to streptomycin (Stenderup, 1954).

\section{Summary}

Eight cases are reported of infection of the urinary tract in which streptomycin-dependent bacteria developed during treatment with streptomycin.

In all cases (in some cases not until days after withdrawal) the streptomycin-dependent strain disappeared after the withdrawal of the drug. In one case the infection was aggravated when administration of streptomycin was resumed.

\section{REFERENCES}

Dufourt, A., Brun, J., Viallier, J., and Kalb, J. C. (1951). Rev. Tuberc., Paris, 5 ser, 15, 1.

Jensen, K. A., and Kjær, I. (1948). Acta path. microbiol. scand., 25, 146.

Kushnick, T., Randles, C. I., Gray, C. T., and Birkeland, J. M. (1947). Ścience, 106, 587.

Miller, C. P., and Bohnhoff, M. (1947). Ibid., 105, 620.

Maine, T. F., and Finland, M. (1948). J. Bact., 56, 207.

Stenderup, A. (1954). Acta path. microbiol. scand., 3 i, 161.

Yegian, D., Budd, V., and Vanderlinde, R. J. (1949). J. Bact., 58, 257. 